\title{
Substance abuse and psychological well-being of South African adolescents
}

\author{
Maretha Visser \\ Department of Psychology, University of Pretoria, Brooklyn, 0002, South Africa \\ maretha.visser@up.ac.za \\ Leigh-Anne Routledge \\ Department of Psychology, University of Pretoria, South Africa
}

This research aimed at identifying patterns of substance abuse among South African adolescents and exploring the relationship between psychological well-being and substance abuse. Psychological well-being was assessed with selected items of the Bar-On EQ-I and Diener's Life Satisfaction scale. Self-reported substance abuse patterns of 1918 learners between the ages of 12 and 19 from 13 schools in Tshwane were recorded. Schools were selected to represent the population composition of the area. It was found that substance abuse (current alcohol use, excessive alcohol use, and use of illicit drugs) increased with age and that nearly twice as many males as females abused substances. Language group was found to be a determining factor with regard to current and excessive use of alcohol; however, it was not found to influence drug use. An analysis of variance showed that adolescents who used drugs had significantly lower levels of psychological well-being and life satisfaction. The same was not found for excessive use of alcohol. The results can contribute to a better understanding of substance use behaviour and to identifying adolescents who may be at risk of abuse.

Keywords: adolescent substance abuse; excessive alcohol use; illicit drug use; life satisfaction; psychological well-being; risk behaviour

Young people are often enticed by drug lords to become pedlars and consumers of illegal substances. We must help empower them to become part of the solution instead of the problem. (Nelson Mandela, quoted in Department of Health, 1999, p. 1)

Substance abuse is an important social and health problem in almost all of the countries in the world including South Africa. History has shown that, when a country experiences widespread and dramatic socioeconomic changes, these frequently resound within the sphere of alcohol and drug intake (Rocha-Silva, 1998; Simon, 1998; Tucker $\&$ Scott, 1992). Although South Africa has been a democracy for more than 10 years now, the country is still undergoing transition and it seems that various substancerelated problems have developed and escalated during this period of transition (Pluddemann et al., 2004; Richter et al., 2006). The current profile of substance abuse 
among South African adolescents in many ways mirrors the trends found globally among adolescents (Parry, Myers, et al., 2004). However, over the past few years the HIV/AIDS epidemic has captured much attention and many resources, contributing to less of a focus on research and preventive interventions aimed at adolescents with regard to substance abuse.

Data from around the world suggest that substance use often starts between the ages of 14 and 15 years (Richter et al., 2006). Although numerous factors can account for adolescent substance use, the predominant reason seems to involve the fact that adolescence is a period of transition, in which individuals seem to be more impulsive, reckless, and non-conforming than during other developmental stages of their lives (Madge \& Harvey, 1999). Many adolescents engage in substance use activities, which they do perceive as risky, but as somehow acceptable within their peer groups. As a result, risk behaviours (including substance abuse) during the adolescent years are of major concern because they are associated with increased risk for injury, interpersonal violence, crime, high-risk sexual behaviour, suicide, academic difficulties, and school drop-out (Flisher, Ziervogel, \& Chalton, 1996; Stoelb, 1998; Sutherland \& Shepherd, 2001). Substance abuse can impact on the rest of their lives. Co-morbidity of substance abuse and other psychological disorders appears to be common among adolescents and young adults. Co-morbid adolescents have a more chronic course, greater impairments in global role functioning, and poorer prognoses, and tend to be less responsive to treatment compared with single disorder cases (Beitman et al., 2001). As a result, the financial burden experienced by adolescents who suffer from both substance-use disorders and poor psychological well-being is overwhelming. According to findings from the South African Community Epidemiology Network on Drug Use (SACENDU) projects, these costs are on the increase, as can be seen by the greater demand for substance-abuse treatment services for adolescents (Parry et al., 2002).

\section{SUBSTANCE ABUSE}

Substance abuse refers to the improper, excessive, irresponsible, or self-damaging use of addictive substances (Reber \& Reber, 2001). The Diagnostic and Statistical Manual of Mental Disorders (DSM-IV-TR) (APA, 2000) defines substance abuse as a maladaptive pattern of substance use leading to clinically significant impairment or distress, manifest by one or more of the following symptoms within a 12-month period: recurrent substance use in situations that cause physical danger to the user, or in the face of obvious impairment in school or work situations, or despite resulting social, interpersonal, or legal problems. The National Drug Master Plan (Department of Health, 1999) maintains that the term substance abuse includes both the misuse and abuse of legal substances such as nicotine, alcohol, over-the-counter drugs, prescribed drugs, alcohol concoctions, indigenous plants, solvents, and inhalants, as well as the use of illicit drugs. Simply put, substance abuse refers to any substance that, when taken by a person, modifies perception, mood, cognition, behaviour, or motor functions (APA, 2000). 
Most researchers agree that any substance use among children and adolescents up to the age of 16 years (18 years in South Africa) constitutes a form of abuse (Richter et al., 2006). The reasons for this are threefold. First, adolescents are still growing at this age and therefore their nervous systems may be particularly susceptible to the negative effects of drugs. Second, the use of alcohol and cigarettes is not legal for adolescents, and therefore they are exposed to potential problems with the law, at school, or with their families. And finally, substance use in children and adolescents has been linked to other serious behavioural problems, such as truancy, school drop-out, delinquent activities, and precocious sexual activities (Newcomb \& Bentler, 1989). Therefore, in adolescence, all substance use is legally defined as substance abuse.

Despite ongoing research, which provides information about the devastating effects substance abuse has on individuals, families, and communities, adolescents continue to abuse substances. It was found that $24 \%$ of adolescents in the United States of America (USA) admitted to using substances during the 2002/2003 school year. This remained consistent with the trend of the past five years (Williams, 2004). A British survey carried out by the National Centre for Social Research found that $45 \%$ of 15 year olds had tried using substances at some point in their lives, and that $36 \%$ had used substances in the past year (Williams, 2004). Research in South Africa has also found that substance abuse among adolescents is one of the most significant health and social problems (Flisher, Parry, Evans, Lombard, \& Muller, 2003).

\section{Alcohol use}

South African statistics show that alcohol is the dominant substance of abuse (Parry et al., 2002). Though different sample populations and definitions of alcohol use and abuse were used, the major studies done amongst South African adolescents during the past 15 years found increased risk proneness with regard to alcohol-related problems. Due to different definitions and contexts of research, key findings from various studies about the prevalence of substance abuse are highlighted:

(a) Flisher, Ziervogel, Chalton, Leger, and Robertson (1993) found that 53\% of a sample of 7340 learners from 16 secondary schools in the Cape Peninsula, from both historically advantaged and disadvantaged communities, had previous experience of drinking alcohol.

(b) Rocha-Silva, De Miranda, and Erasmus (1995) found that $42 \%$ of the respondents, who were black youths from urban and rural areas, reported that they had had a drink of alcohol at some time in their lives, while $34 \%$ of the sample reported current drinking patterns (in the 12 months preceding the survey). Current drinkers were more common in the urbanised areas, and amongst males. They also found that regular use of alcohol (at least once a week) was common among the older age group (18 to 21 years). 
(c) A representative survey involving 6000 learners in grades 8 and 11 in 39 schools in Cape Town showed that the highest prevalence of substance use was in the grade 11 male group, where 50\% reported current alcohol use and $36 \%$ males and $19 \%$ females reported binge drinking (more than five drinks per occasion) during the two weeks prior to the study (Flisher, Parry, Evans, Lombard, \& Muller, 1998, 2003).

(d) From a representative sample of 38 schools from Durban, it was found that 53\% males and 29\% females in grade 11 reported binge drinking during the two weeks prior to the study (Bhana, Flisher, \& Parry, 1998).

(e) In a sample of 300 young people in Gauteng and Limpopo provinces, $50 \%$ of the young people in the rural area reported current alcohol use. In the urban area, females reported current use of wine $(68 \%)$ and males used mainly malt beer $(80 \%)$, hard liquor, and home-made liquor (67\%) (Weir-Smith, 2001).

(f) The Youth Risk Behaviour survey reports that $34 \%$ of young people between the ages of 14 and 18 years drink alcohol occasionally, and 24\% drink alcohol at least once a week, while $3 \%$ reported daily alcohol use (Reddy et al., 2002).

The South African data reported in these studies show a progressive increase in the use of alcohol during the past decade. Although alcohol was cited as the second most common primary substance of abuse after cannabis within treatment centres, excessive drinking is the most common form of substance misuse among adolescents of both genders. In keeping with international findings, it appears on the one hand that the prevalence rate for alcohol use, and especially excessive drinking, is much higher among males (Parry, Myers, et al., 2004). On the other hand, Reddy et al. (2002) report that the gender gap is narrowing, especially in the white population. In both genders, the prevalence of alcohol use increases with age (Parry, Morojele, Saban, \& Flisher, 2004; Parry, Myers, et al., 2004). White youth had the highest rates of weekly drinking (32\%), followed by Indian $(26 \%)$ and African $(23 \%)$ respondents, while coloureds had the lowest rates (19\%) (Reddy et al., 2002). The Community Agency for Social Enquiry (CASE) (2002) also found that more white young people than African young people indicated using alcohol, although more African respondents experienced alcohol use as a problem in their community. Rocha-Silva, Mokoko, and Malaka (1998) reported that heavy drinking was higher among African males.

\section{Drug use}

There is a paucity of information on the extent of drug use amongst South African young people. The available research focuses on small or specific populations. Young people were found to be disproportionately involved in drug use, with drug use often beginning in the early teenage years (Richter et al., 2006). A number of studies point to cannabis (dagga) as the most common substance used among South African youth, especially among males (Weir-Smith, 2001). Rocha-Silva et al. (1995) found that 3.8\% 
of the black youth in their research group admitted that they had used cannabis, while Flisher et al. $(1998,2003)$ reported current cannabis use by $3 \%$ of the grade 8 and $16 \%$ of the grade 11 males in their study involving 39 Cape Town schools. In a sample of 460 primary school children (grades 6 and 7) aged 11 to 16 years, Visser (2003) found that $4 \%$ reported smoking cannabis.

The prevalence rate for use of other illicit drugs varied from $2 \%$ of a sample reporting that they smoke 'white pipe' (mandrax and dagga) (Rocha-Silva et al., 1995) to $5.7 \%$ of grade 11 males who reported usage of mandrax (Flisher et al., 1998, 2003). Other substances abused were sniffing solvents (7.4\%) (Rocha-Silva et al., 1995) and over-the-counter medicines - most popular among females (Weir-Smith, 2001). These findings mirror past South African as well as international substance abuse trends (Parry, Myers, et al., 2004). Drug use was found to be more common among white youth (Birkett, 2001; CASE, 2002). Reddy et al. (2002) report that 34\% of white adolescent respondents reported that they had used drugs some time in their lives, compared to $20 \%$ of the Indian, $19 \%$ of the coloured and $10 \%$ of the African youth. In all the population groups except whites, males were twice as likely as their female counterparts, to use illicit drugs.

Much of the research conducted in South Africa to date focuses on obtaining epidemiological data. In this study, the relationship between substance abuse and psychological well-being will be explored as well.

\section{Psychological well-being and substance abuse}

Psychological well-being is a relatively broad concept referring to a good or satisfactory condition of existence, a state characterised by health, happiness, and prosperity. The term psychological well-being is often used interchangeably with the term mental health. It refers to functioning at a high level of behavioural and emotional adjustment and adaptiveness, and not merely an absence of illness (Reber \& Reber, 2001). Individuals with higher than average psychological well-being are regarded as more successful in meeting environmental demands and pressures, while a deficiency in psychological well-being can mean a lack of success and the existence of emotional problems (Bar-On, 1988). According to Bar-On (1988, 2000), the most important reliable factorial components of psychological well-being are self regard, interpersonal relationships, independence, problem solving, assertiveness, reality testing, stress tolerance, self-actualisation, and happiness. However, for the purpose of this research, the term psychological well-being refers to one's overall psychological health.

The relationship between substance abuse and psychological well-being is a complex one. Both variables are affected by the interaction of biological, psychological, and social factors (Green \& Shellenberger, 1991). Although this study focuses predominantly on the individual, behaviour cannot be seen in isolation from the larger social, political, cultural, and economic environment or macro-level in which it occurs (Bronfenbrenner, 1979). Risk behaviour such as substance abuse can therefore be related to individual 
psychological factors such as self-esteem, locus of control, need for acceptance, anxiety levels, sensation seeking, and eagerness to act like adults (Shiel, 1999). However, substance abuse is also closely linked to social and community factors such as access and exposure to substances, social norms that tolerate risk behaviour, peer pressure, socioeconomic status, educational opportunities, social support, and involvement with a social network. In this research, the relationship between substance abuse and psychological well-being will be explored as an individual state influenced by multiple factors.

\section{METHOD}

The objectives of the research were twofold: (a) to describe the extent of substance abuse among school-going adolescents in Tshwane, and (b) to relate indicators of psychological well-being to substance abuse. A self-report questionnaire was completed by a large sample of adolescents to investigate the relationships between variables.

\section{Research participants}

The respondents consisted of 1918 adolescents between the ages of 12 and 19 years who attended schools in Tshwane. The Education Department identified 13 schools as representative of the population of the area to participate in a peer support project. One school was from a previously white residential area, two were from a traditionally coloured area, two from a predominantly Indian area, and eight schools from a mainly black residential area. The baseline data of the peer support project were used in this research. Within each school, a random sample of a class per grade was used as the sample of respondents. A randomly selected class of learners was used instead of randomly selected learners, because of an agreement with the principals of the schools not to disrupt the normal school day too much. The sample was therefore not completely representative, which limits the generalisability of the findings.

\section{Data-gathering instruments}

The data-gathering instruments consisted of two psychometric scales and questions about behaviour related to substance abuse.

\section{Psychological well-being}

To assess psychological well-being, selected items of the Bar-On Emotional Quotient Inventory (EQ-I) were used. The EQ-I developed from research done by Bar-On (1988), in which factors related to psychological well-being were identified to formulate an operational definition of psychological well-being. The EQ-I assesses various aspects of 
psychological well-being such as self regard, interpersonal relationships, independence, problem solving, assertiveness, reality testing, stress tolerance, self-actualisation, and happiness. Using the data of 2000 subjects from seven countries, Bar-On (2000) reports a Cronbach's alpha internal consistency coefficient of 0.76 for the scale as a whole. High correlations were obtained with various measures of personality and estimates of effective coping with the pressures and demands of daily life (Bar-On, 2000).

In the present study, 22 items from the self-regard, interpersonal relationships, and happiness sub-scales of the EQ-I were selected as a brief indicator of feelings of psychological well-being. These items were chosen to reflect aspects of intrapersonal, interpersonal, and general mood components of psychological well-being (Bar-On, 2000). Responses were coded on a 3-point Likert-type scale: 'agree', 'in between', and 'disagree'. In a factor analysis using the data of the 1918 respondents in the present study, one general factor was extracted. Factor loadings varied from 0.27 to 0.59 . A Cronbach's alpha coefficient of 0.794 indicated an acceptable internal consistency between the items on the scale for this sample (Kerlinger \& Lee, 2000).

\section{Life satisfaction}

Diener's Life Satisfaction scale, consisting of five items, assesses the cognitive component of a subjective experience of well-being (Diener, Sandvik, \& Pavot, 1991). Life satisfaction can be defined as a global evaluation of a person's life. Each person constructs a standard for him- or herself of what an acceptable life is and compares his or her own life with this standard. Life satisfaction is a subjective value (Diener, Larsen, $\&$ Griffen, 1985). In a few studies done with students, Diener et al. (1985) found that the scale measures a single factor of global life satisfaction accounting for 66 per cent of the variance. A high internal consistency with a Cronbach's alpha coefficient of 0.87 and a test-retest correlation coefficient of 0.82 with a two-month interval were reported (Diener et al., 1985). Similar findings have been reported by Pavot and Diener (1993) and Yardley and Rice (1991). In validity studies, it was found that the scale correlated highly with other wellness questionnaires and clinical reports (Diener et al., 1985).

In the present study, the responding was simplified by using a three-point Likert-type scale. In previous research using a similar sample of 856 adolescents, item-scale correlations varied between 0.51 and 0.70 and a Cronbach's alpha coefficient of 0.654 was reported (Visser, 2001). In this study, the Cronbach's alpha coefficient for the data of 1889 learners was 0.59 , which is rather low.

\section{Reported substance abuse}

Substance abuse was assessed using self-reported behaviour with regard to alcohol and drug use. Questions were developed for the study, based on the guidelines put forward by the Center for Substance Abuse and Mental Health Services (Kumpfer, Shur, Ross, Bunnell, Librett, \& Millward, 1993). The following questions were asked: 
(a) Did you drink alcohol during the past 30 days - more than just a sip? (Current alcohol use)

(b) Did you drink five or more drinks with alcohol on one day during the past 30 days? (Excessive alcohol use or binge drinking)

(c) Have you used drugs such as dagga (zol), cocaine, crack, mandrax (white pipe), LSD during the past 30 days? (Illicit drugs use)

A self-report questionnaire reflects on the experiences of the respondents. Self-report questionnaires are particularly useful in enhancing honest reporting of risk behaviours without revealing confidential information to a teacher or research assistant in an interview situation (Turner, Lessler, \& Gfoerer, 1992). In spite of using various techniques to minimise circumstances that might impair the reliability of the responses, such as promises of anonymity and confidentiality, there is always a possibility of overor under-reporting in self-report questionnaires. The possibility of problems in reliability seems increased when the behaviours reported on are labelled as deviant or illegal (Tarter, 2002). Even so, findings indicate that in general the reliability of selfreports is high, with only a small tendency toward over- and under-reporting (Ford, 2001). Perhaps more important, Schwartz and Stack (2003) found that the type of response scale that is used, the response options, and the order and presentation of questions can all influence the levels of honesty when reporting high-risk behaviour such as substance use.

\section{Data collection procedure}

Permission to conduct the research as part of a broader study on the effectiveness of peer support in schools was obtained from the Education Department. The principals, school governing bodies, and guidance teachers of the respective schools were informed about the project and asked whether their schools would voluntarily participate in the peer support project. The parents were informed about the research at a parents' meeting due to the fact that many learners were younger than 18 years. Parents were given the option to withdraw their children from the study. Arrangements were made with the principals of each school to collect data at the schools during a specific week. The guidance teachers selected one class from each grade group at random and arranged that the learners of the class complete the questionnaire during their guidance period.

In the guidance classes, a research assistant explained the purpose of the broader peer support project and how these baseline data could assist in the development of the project. The research assistant asked the learners whether they would be willing to participate in this research. Those who were not willing to participate were given the options of either leaving the room or just not completing the questionnaire. Almost all the learners completed the questionnaire, since there were very few uncompleted or unfinished questionnaires. 
The questionnaire was given in English, since the majority of the participants were fluent in English. A research assistant was present to deal with questions or to translate words that the participants did not understand. Questionnaires were completed anonymously, and any information that could link the adolescents to their responses was kept confidential. Completed questionnaires were placed in a sealed box to be opened at another venue by the researchers.

\section{Data analysis}

To investigate substance-use patterns, chi-square analyses (Neuman, 1997) were performed to express the strength of the relationship between substance abuse and age, gender, and language group. Because of the large sample size, normal statistics may show significant differences while the effect of the difference in the mean scores is very small. This can be corrected by calculating the effect size, in the form of the contingency value. According to Cohen (1988), a contingency value of less than 0.1 indicates a small effect, 0.3 a medium effect, and more than 0.5 a large effect.

Scale scores were calculated for the Psychological well-being and Life Satisfaction scales. A one-way analysis of variance (ANOVA) was conducted with psychological well-being as the dependent variable and gender, age, language, excessive alcohol use, and drug use as the independent variables. (Current alcohol use was not used as a variable in this analysis, because all learners who used alcohol excessively were also part of learners who currently used alcohol.) An ANOVA serves to highlight how well a set of independent variables explains a dependent variable (Neuman, 1997). The interaction between the two variables reflecting substance use was also investigated in a factorial analysis of variance. These results were not reported here because the results were not significant. The same procedure was followed with life satisfaction as dependent variable. The partial eta squared is used as an indication of effect size. Effect size indexes for the analysis are the following: less than 0.01 indicates a small effect size, a value of 0.1 indicates a medium effect size, and 0.25 and more indicates a large effect size (Cohen, 1988).

\section{RESULTS}

\section{Biographical characteristics of the respondents}

The sample consisted of $44 \%$ males and $56 \%$ females between the ages of 12 and 19 years. The age distribution is given in Table 1. The largest proportion of the sample $(55 \%)$ was between 15 and 17 years old. 
Table 1. Age distribution

\begin{tabular}{|ll|}
\hline Age & $\boldsymbol{N}(\mathbf{\%})$ \\
\hline 12 to 13 years & $146(7)$ \\
14 years & $285(15)$ \\
15 years & $323(17)$ \\
16 years & $397(21)$ \\
17 years & $317(17)$ \\
18 years & $230(12)$ \\
19 years & $44(2)$ \\
Missing values & $176(9)$ \\
\hline
\end{tabular}

The majority of the respondents (77\%) spoke African languages; the largest groups were Sepedi $(25 \%)$, isiZulu (15\%), Setswana (13\%), and Tsonga (12\%). Afrikaansspeaking adolescents formed $9 \%$ of the sample and English-speaking adolescents $7 \%$. Another $7 \%$ did not complete the question. Thus, the sample was fairly representative of the community, although the black group was over-represented. Schools in different residential areas were selected to obtain a representation of learners in various language groups, but it was found that black learners also attended schools in traditionally white, coloured, and Indian residential areas, while the opposite was not true in mainly black communities.

\section{Patterns of substance use}

In this study, $26 \%$ of the respondents reported current alcohol use and $14 \%$ reported excessive or binge drinking (more than five alcoholic drinks per occasion) in the past 30-day period, while $7.5 \%$ reported the use of illicit drugs such as cannabis (dagga), cocaine, crack, mandrax, or LSD. While the excessive alcohol users were a sub-group of the current alcohol users, there were 5\% learners who used alcohol excessively as well as drugs, while $12 \%$ used alcohol excessively but not drugs, and 3\% used drugs but did not use alcohol excessively. Therefore, in analysing the data, the interaction effect needed to be explored. Substance use patterns were investigated in different age, gender, and language groups.

\section{Substance abuse and age}

Chi-square analyses (Table 2) showed significant relationships between age and all three categories of substance use $(p<0.001)$. Because of the large sample size, the contingency value or effect size was also calculated. It was found that the differences had a small effect in this sample $(0.1<C<0.3)$. The results indicated that current alcohol use, excessive alcohol use, and use of illicit drugs increased with age. For example, where at age 13 only $13 \%$ of the adolescents reported alcohol use, at age 19 the percentage had risen to $45 \%$. Where no 13 -year-old adolescents reported drug use, $23 \%$ of their 19-year-old counterparts did so. 


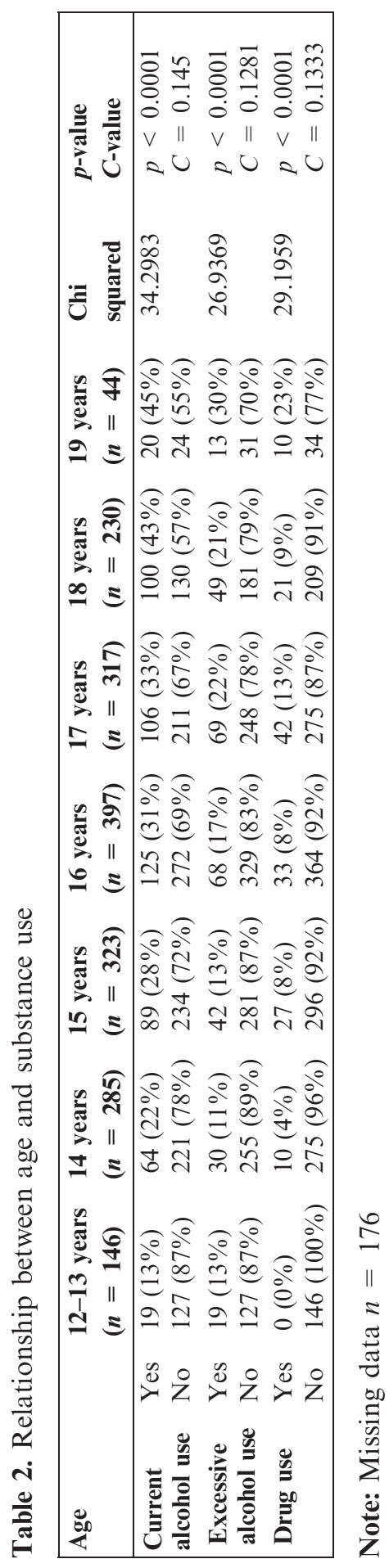




\section{Substance abuse and gender}

The results of this study revealed large differences in the substance use between genders: $41 \%$ of the male adolescents compared to $23 \%$ of the females reported current alcohol use. The same pattern was found for excessive alcohol and drug use (Table 3). Therefore males tended to be far more prone to substance use than females. These differences were statistically significant $(p<0.001)$ and had a small to medium effect on all three variables $(0.1<\mathrm{C}<0.3)$.

Table 3. Relationship between gender and substance use

\begin{tabular}{|llllll|}
\hline Gender & & $\begin{array}{l}\text { Male } \\
(n=808)\end{array}$ & $\begin{array}{l}\text { Female } \\
(n=1006)\end{array}$ & Chi square & $\begin{array}{l}\boldsymbol{p} \text {-value } \\
\boldsymbol{C} \text {-value }\end{array}$ \\
\hline Current alcohol use & Yes & $330(41 \%)$ & $234(23 \%)$ & 64.5829 & $p<0.0001$ \\
& No & $478(59 \%)$ & $772(77 \%)$ & & $\mathrm{C}=0.1858$ \\
Excessive alcohol use & Yes & $190(24 \%)$ & $118(12 \%)$ & 44.6141 & $p<0.0001$ \\
& No & $618(76 \%)$ & $888(88 \%)$ & & $\mathrm{C}=0.1548$ \\
Drug use & Yes & $121(15 \%)$ & $32(3 \%)$ & 81.5447 & $p<0.0001$ \\
& No & $687(85 \%)$ & $974(97 \%)$ & & $\mathrm{C}=0.2072$ \\
\hline
\end{tabular}

Note: Missing data $n=104$

\section{Substance abuse and language}

Substance use of learners of the largest language groups was compared (Table 4). Different language groups showed significantly different patterns of current alcohol use, excessive drinking, and drug abuse $(p<0.05)$, although the effect size was small (C $<0.1)$. English- and Tsonga-speaking adolescents reported the lowest rates of current drinking behaviour (23\% and $25 \%$ respectively), while $36 \%$ of the Afrikaans and Setswana adolescents reported current alcohol use. The English- and Tsonga-speaking adolescents also had the lowest rates of excessive alcohol use, while the other African language speakers, isiZulu, Setswana, and Sepedi speakers, had the highest rates of excessive alcohol use. The Setswana speakers had the highest level of reported drug abuse (14\%), while the Zulu speakers had the lowest level $(6 \%)$. 


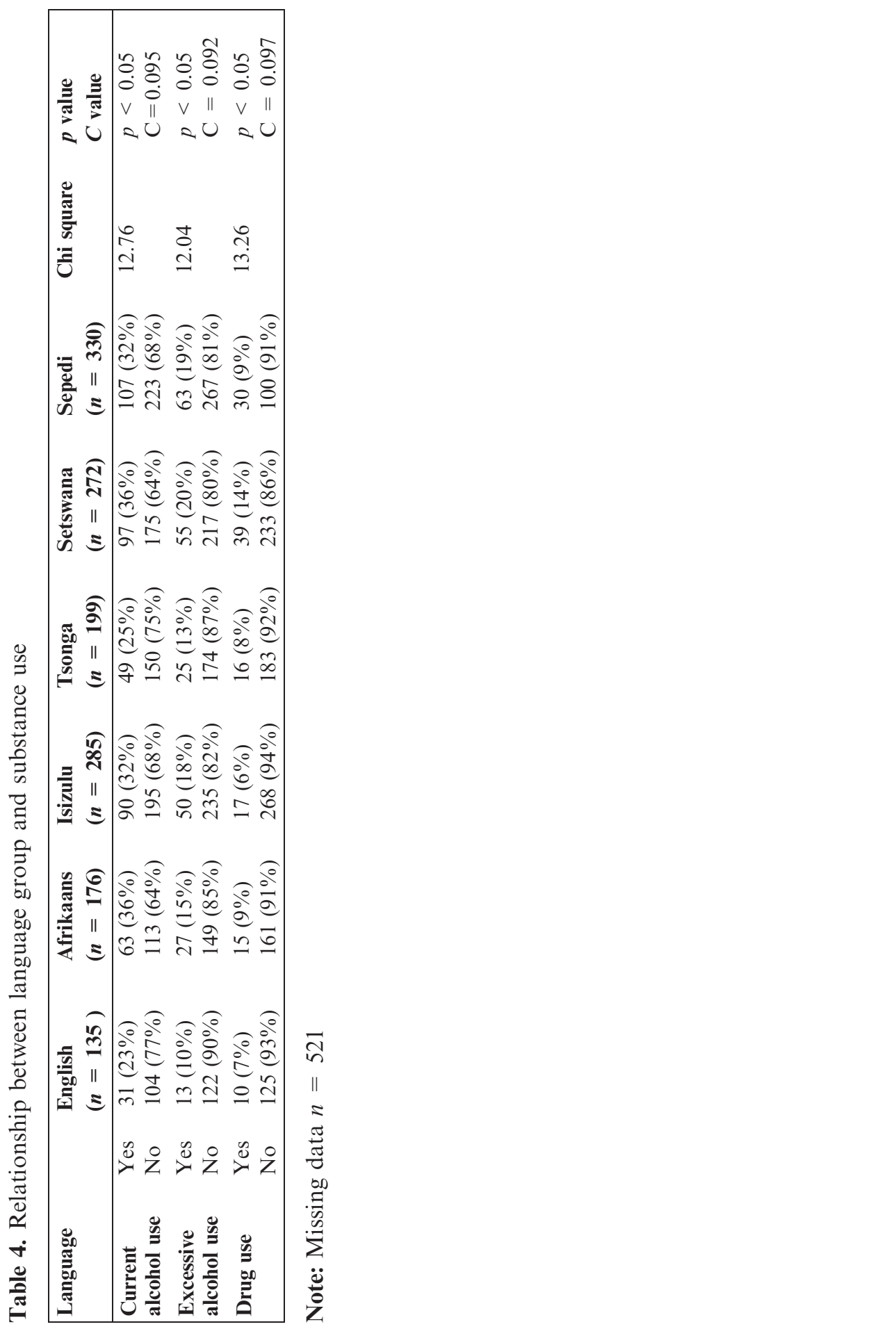




\section{Psychological well-being and substance abuse}

Scale scores for psychological well-being and life satisfaction were calculated. It was observed that the majority of the respondents scored high on the psychological well-being scale. The mean score was 51.86 on a scale ranging from 22 to 66 (a high score meaning high levels of well-being). The same was found for the life satisfaction scale. The average life satisfaction score of 8.63 was found on a scale ranging from 5 to 15 (where a low score indicated more life satisfaction). There was a negative correlation of 0.4 between the two scales that could explain some of the similarities in the two sets of results.

An ANOVA was conducted with psychological well-being as the dependent variable and gender, age, language, excessive alcohol use, and drug use as the independent variables (Table 5). (Because excessive alcohol use implies alcohol use, the alcohol use variable was left out in this analysis.) To investigate possible interaction effects between excessive alcohol use and drug use a factorial ANOVA was done, with main effects and interactions $F(16,1405)=2.6(p<0.001)$. No interaction effects were significant $(p<0.05)$. Therefore only the one-way analysis is reported in Table 5.

Table 5. Factors related to psychological well-being (One-way ANOVA)

\begin{tabular}{|llrllll|}
\hline Variable & & N & Mean & F & $p$ & $\begin{array}{l}\text { Effect size } \\
\text { (Partial eta } \\
\text { squared) }\end{array}$ \\
\hline Gender & Male & 514 & 52.0 & 0.77 & 0.38 & \\
& Female & 703 & 52.1 & & & \\
& 14 & 300 & 52.7 & 1.17 & 0.32 & \\
& 15 & 237 & 51.6 & & & \\
& 16 & 269 & 52.4 & & & \\
& 17 & 225 & 51.8 & & & \\
Language & English & 129 & 53.2 & 2.64 & $0.02 *$ & \\
& Afrikaans & 169 & 51.4 & & & \\
& isiZulu & 231 & 51.9 & & & \\
& Tsonga & 175 & 51.0 & & & \\
& Setswana & 234 & 52.7 & & & \\
Excessive & Sepedi & 279 & 52.2 & & & \\
alcohol use & Yes & 240 & 50.7 & 5.21 & $0.02 *$ & \\
Drug use & Yes & 1022 & 52.3 & & & \\
& No & 126 & 49.9 & 7.03 & $0.008^{*}$ & $0.006^{* * *}$ \\
& No & 1294 & 52.3 & & & \\
\hline
\end{tabular}

Note: Cases with missing values were excluded from the data analysis.

$*=p<0.05$

$* *=$ small effect size

$* * *=$ medium effect size 
Table 5 shows that psychological well-being is significantly affected by excessive alcohol use $(p<0.05)$ and drug abuse $(p<0.01)$, though the effect size was small. Respondents who reported excessive alcohol and drug use had significantly lower levels of psychological well-being. English- and Tswana-speaking respondents were found to have higher levels of psychological well-being than Afrikaans and Tsonga speakers $(p<0.05)$, though the effect size was also small.

The same procedure was followed to link life satisfaction scores with the independent variables (Table 6). The interaction effects between excessive alcohol use and drug use were also investigated using a factorial ANOVA with main effects and interactions $F(16,1407)=2.08(p<0.01)$, but again no interaction effects were significant $(p<0.05)$. The results of the one-way ANOVA are therefore given in Table 6.

Table 6. Factors related to life satisfaction (One-way ANOVA)

\begin{tabular}{|c|c|c|c|c|c|c|}
\hline Variable & & $N$ & Mean & $F$ & $p$ & $\begin{array}{l}\text { Effect size } \\
\text { (Partial eta } \\
\text { squared) }\end{array}$ \\
\hline \multirow[t]{2}{*}{ Gender } & Male & 517 & 8.8 & 2.1 & 0.15 & \\
\hline & Female & 704 & 8.6 & & & \\
\hline \multirow[t]{6}{*}{ Age } & 14 & 302 & 8.5 & 1.36 & 0.24 & \\
\hline & 15 & 237 & 8.7 & & & \\
\hline & 16 & 270 & 8.7 & & & \\
\hline & 17 & 225 & 8.8 & & & \\
\hline & 18 & 159 & 8.7 & & & \\
\hline & 19 & 38 & 9.2 & & & \\
\hline \multirow[t]{6}{*}{ Language } & English & 129 & 9.2 & 4.22 & $0.001^{*}$ & $0.017 * *$ \\
\hline & Afrikaans & 170 & 9.0 & & & \\
\hline & isiZulu & 231 & 8.7 & & & \\
\hline & Tsonga & 176 & 8.8 & & & \\
\hline & Setswana & 234 & 8.5 & & & \\
\hline & Sepedi & 281 & 8.5 & & & \\
\hline \multirow{4}{*}{$\begin{array}{l}\text { Excessive } \\
\text { alcohol use } \\
\text { Drug use }\end{array}$} & Yes & 195 & 8.6 & 0.97 & 0.33 & \\
\hline & No & 1026 & 8.7 & & & \\
\hline & Yes & 127 & 9.2 & 4.44 & $0.04 *$ & $0.004 * *$ \\
\hline & No & 1114 & 8.6 & & & \\
\hline
\end{tabular}

Note: Cases with missing values were excluded from the data analysis.

$*=p<0.05$

$* *=$ small effect size.

In this analysis, drug abuse was related to levels of life satisfaction $(p<0.05)$ although the effect size was small. Adolescents who used drugs were more likely to experience less life satisfaction, while those who abstained from using drugs were more likely to be more satisfied with their lives. Life satisfaction scores were not significantly related to excessive alcohol use. Life satisfaction was also related to language group $(p<0.001)$ 
with a medium effect size. Setswana and Sepedi speakers reported more satisfaction in life than English speakers.

\section{DISCUSSION}

The pattern of substance abuse observed in this study seems to be consistent with local and international patterns (Parry, Myers, et al., 2004; Pluddemann et al., 2004; Weinberg, 2001; Williams, 2004). The results indicate that $26 \%$ of the respondents used alcohol during the past 30 days and $14 \%$ used alcohol excessively. Alcohol use was found to be less in the current study than in a similar school sample in the research of Flisher et al. (2003), but corresponded with findings of Reddy et al. (2002). The 7.5\% of respondents who indicated use of various types of illicit drugs seems to be less than the grade 11 group in the Cape Town sample (Flisher et al., 2003) where separate questions were asked about various types of drugs. Of concern is the fact that all the respondents were 19 years and younger. There is evidence that adolescents who start drinking, smoking, and using cannabis at an early age are more likely to turn to 'hard' drugs than those who start later in life (Degenhardt, Hall, \& Lewinsky, 2001). Adolescents involved with any form of substance use are also more likely to be involved in other risky behaviours and they are also more likely to have academic problems (Flisher et al., 1996; Weinberg, 2001).

The findings in the study are consistent with past research, as many researchers have suggested that the average age for starting the use of substances is around 11 to 14 years, and that substance use increases with age during the adolescent years (Flisher et al., 2003; Parry, Morojele, et al., 2004; Rocha-Silva et al., 1998). This research also shows that males are almost twice as likely to engage in substance use as females are: $41 \%$ of the males reported current alcohol use compared to $23 \%$ of the females. This pattern was also found for excessive or binge drinking and taking illicit drugs. This pattern has consistently been found in other research (Parry, Myers, et al., 2004; Pluddemann et al., 2004; Rocha-Silva et al., 1995), except for white respondents in the study of Reddy et al. (2002) where the gender gap was found to be narrowing. Gender differences in substance abuse may be related to social roles and social expectations of male and female behaviour.

Culture and cultural identification have been shown to affect levels of substance use (Glendinning, Shucksmith, \& Hendry, 1994; Kaplan \& Sadock, 2003). Substance use can be regarded as a social act embedded in a context of values, attitudes, and conceptions of reality, related to cultural beliefs. In this research, language group was used as a variable. It was found that English- and Tsonga-speaking adolescents had the lowest level of substance abuse, while Setswana speakers were amongst the highest users in all the categories. These results indicate that patterns of substance abuse can be influenced by community factors. Previously it was found that white respondents were more often current drinkers of alcohol (CASE, 2002; Parry, Morojele, et al., 2004; Reddy et al., 2002), while heavy drinking was more prevalent in the African context (CASE, 2002; Rocha-Silva et al., 1998). 
Besides the social indicators, this research also established a link between psychological well-being and substance abuse. One-way analyses of variance showed that adolescents who reported excessive alcohol use and drug use had significantly lower levels of psychological well-being and adolescents reporting drug use had lower levels of life satisfaction than their peers. The effect size of the results was small, indicating that significance could have been due to the large sample size. The diverse relationships between language, substance abuse, and life satisfaction show that these relationships found are complex relationships with many other variables also impacting on the variables. The relationship between substance abuse and psychological wellbeing can possibly be explained by Barlow and Durand's (1999) theory that individuals often use substances as a means to escape when life poses too many challenges. In explaining this, though, the 'chicken or egg' question plagues research. Whether or not poor psychological well-being leads to drug use, in an effort to escape painful feelings, or whether drug use leads to deterioration in overall psychological well-being remains an area of blurred opinions. However, the prior option seems to have gained more weight in recent years (Armstrong \& Costello, 2002). This research shows that a measure of psychological well-being can be used to identify adolescents who may possibly be at risk of abusing substances in a risk-taking context.

These findings suggest that individual factors such as psychological well-being and social factors such as age, gender, and language group have an effect on adolescents' level of substance use. The interaction between psychological and social factors of the adolescents' environment and the impact on substance use behaviour are therefore acknowledged (Bronfenbrenner, 1979). The social and cultural contexts of the adolescent are made up of the peer group, access and exposure to substances, social norms, socioeconomic status, education, and social support networks.

The period of adolescence lends itself to egocentrism and a sense of being invulnerable, encouraging adolescents to take risks (Williams, 2004). One can therefore see how being an adolescent, and especially being a male surrounded by societal stereotypes and pressures, can contribute to psychological difficulties and risk-taking behaviour. Being part of certain cultures and communities where there is higher exposure to substance use can also increase the likelihood of becoming involved in substance abuse. All these contexts need to be taken into account to understand the nature and aetiology of adolescent substance abuse.

\section{LIMITATIONS OF THE STUDY}

The study used self-reported data and relied on respondents' self-knowledge and subjective experience of situations. This may impact on the accuracy and validity of the results (Tarter, 2002). Research has shown, however, that even when illegal behaviours are reported on, the reliability of self-reports is high, with only a small tendency toward over- and under-reporting (Ford, 2001).

A large sample of 1918 learners from 13 schools was used to gather data. The sample 
of learners was not randomly selected, but a random sample of 65 classes in these schools was used. Schools from different areas were selected in trying to obtain a representative sample of the Tshwane community. The schools were multi-racial and co-educational, and there was no exclusive use of any one language at the schools. As a result, the majority of the sample were African language speakers. The sample is thus not representative of the adolescent school-going population in the area. This implies that the results cannot be generalised, but is suggestive of possible patterns.

Perhaps the most important limitation of the study is the fact that illegal drugs were grouped together, and that no inferences about specific drug use could be generated from this research. The epidemiology with regard to different drugs varies dramatically (Parry et al., 2002). If illicit drugs were, however, separated in the questionnaire, the percentage of respondents reporting use of specific drugs could have been very small, making it difficult to come to any conclusion regarding drug use patterns. This is a limitation of population surveys to study prevalence of less commonly used substances (Parry, Myers, et al., 2004).

\section{CONCLUSION}

By identifying adolescents at risk for substance abuse early, and by providing them with the necessary skills to cope effectively, one can hopefully minimise the amount of stressors these adolescents have at a transitional stage in their lives. The early initiation into substance use, as well as the increase in usage with age, suggests that prevention approaches should be targeting children at a very young age. Similarly, the tendency of a relationship between excessive alcohol use and drug use and psychological well-being, identified in this research, highlights the importance of mental health during the adolescent period. Research results suggest that the onset of psychological disorders usually precedes that of substance abuse (Armstrong \& Costello, 2002). A measure of psychological well-being can perhaps be used to identify adolescents who have low levels of psychological well-being and thus who are at a greater risk of developing substance abuse problems and associated disorders. The vicious tension-reduction cycle of psychological problems leading to substance use and then further psychological deterioration can possibly be changed at an earlier point before adolescents enter rehabilitation centres.

\section{REFERENCES}

American Psychiatric Association (APA). (2000) Diagnostic and statistical manual of mental disorders. Washington, DC: American Psychiatric Association.

Armstrong, T. D., \& Costello, E. J. (2002). Community studies on adolescent substance use, abuse or dependence and psychiatric comorbidity. Journal of Consulting and Clinical Psychology, 7(6), 1224-1239. 
Barlow, D. H., \& Durand, V. M. (1999). Abnormal psychology (2nd ed.). Pacific Grove, CA: Brooks/Cole.

Bar-On, R. (1988). The development of an operational concept of psychological wellbeing. Unpublished doctoral dissertation, Rhodes University, South Africa.

Bar-On, R. (2000). Emotional and social intelligence: Insights from the Emotion Quotient Inventory. In R. Bar-On \& J. D. A. Parker (Eds.), The handbook of emotional intelligence (pp. 363-388). San Francisco: Jossey-Bass.

Beitman, J. H., Adlaf, A. M., Douglas, L., Atkinson, L., Young, A., Johnson, C. J., Escobar, M., \& Wilson, B. (2001). Comorbidity of psychiatric and substance use disorders in late adolescence: A cluster analytic approach. American Journal of Drug and Alcohol Abuse, 27(3), 421-440.

Bhana, A., Flisher, A. J., \& Parry, C. D. H (1998). School survey of substance use among students: Grades 8 and 11 in the Durban metro region. Southern African Journal of Child and Adolescent Mental Health, 11(2), 131-132.

Birkett, C. (2001). Risks, protective factors and coping style in the lives of young people: Findings from a survey of university students' health and well-being. Unpublished master's dissertation, University of Natal, South Africa.

Bronfenbrenner, U. (1979). The ecology of human development. Cambridge, MA: Harvard University Press.

Cohen, J. (1988). Statistical power analysis for the behavioural sciences (2nd ed.). Hillsdale, NJ: Erlbaum.

Community Agency for Social Enquiry (CASE). (2002). Youth 2002: A study of youth in South Africa. A report for the Royal Netherlands Embassy. Johannesburg: CASE.

Degenhardt, L., Hall, W., \& Lewinsky, M. (2001). Alcohol, cannabis and tobacco use among Australians: A comparison of the associations with other drug use and use disorders, affective and anxiety disorders and psychosis. Addiction, 96, 1603-1609.

Department of Health (1999). National drug master plan. Pretoria: Department of Health.

Diener, E., Larsen, R. J., \& Griffin, S. (1985). The satisfaction with life scale. Journal of Personality Assessment, 49(1), 71-75.

Diener, E., Sandvik, E., \& Pavot, W. G. (1991). Happiness is the frequency, not the intensity, of positive versus negative affect. In F. Strack, M. Argyle, \& N. Schwartz (Eds.), Subjective well-being: An interdisciplinary perspective (pp. 119-139). New York: Pergamon Press.

Flisher, A. J., Parry, C. D. H., Evans, J., Lombard, C., \& Muller, A. (1998). Methodology and preliminary results. The South African community epidemiology network on drug use (SACENDU). Paper presented at the 10th National Psychiatric Conference, Johannesburg, South Africa.

Flisher, A. J., Parry, C. D. H., Evans, J., Lombard, C., \& Muller, A. (2003). Substance use by adolescents in Cape Town: Prevalence and correlates. Journal of Adolescent Health, 32, 58-65. 
Flisher, A. J., Ziervogel, C. F., \& Chalton, D. O. (1996). Risk taking behaviour of Cape Peninsula high school students: Part X. Multivariate relationships among behaviours. South African Medical Journal, 86, 1094-1098.

Flisher, A. J., Ziervogel, C. F., Chalton, D. O., Leger, P. H., \& Robertson, B. A. (1993). Risk taking behaviour of Cape Peninsula high-school students: Part IV. Alcohol use. South African Medical Journal, 83, 480-482.

Ford, J. (2001). Substance use and self-reported mental health: The moderating effect of acquaintance use behaviour among adults. Journal of Drug Issues, 31(2), 565-590.

Glendinning, A., Shucksmith, J., \& Hendry, L. (1994). Social class and adolescents' smoking behaviour. Social Science Medical Journal, 38(1), 1449-1460.

Green, J., \& Shellenberger, R. (1991). The dynamics of health and well-being: $A$ biopsychosocial approach. London: Holt, Rinehart \& Winston.

Kaplan, H. I., \& Sadock, B. J. (2003). Synopsis of psychiatry. New York: Williams \& Wilkins.

Kerlinger, F. N., \& Lee, H. B. (2000). Foundations of behavioral research (4th ed.). Fort Worth, TX: Harcourt.

Kumpfer, K. L., Shur, G. H., Ross, J. G., Bunnell, K. K., Librett, J. J., \& Millward, A. R. (1993). Measurements in prevention: A manual on selecting and using instruments to evaluate prevention programs. Center for Substance Abuse and Mental Health Services Administration Technical Report 8. Rockville, MD: US Department of Health and Human Services.

Madge, N., \& Harvey, J. G. (1999). Suicide among the young: The size of the problem. Journal of Adolescence, 22, 145-155.

Neuman, W. L. (1997). Social research methods: Quantitative and qualitative approaches. London: Allyn \& Bacon.

Newcomb, M. D., \& Bentler, P. M. (1989). Substance use and abuse among children and teenagers. American Psychologist, 44, 242-248.

Parry, C. D. H., Bhana, A., Myers, B., Pluddemann, A., Siegfried, N., Morojele, N., Flisher, A. J., \& Kozel, N. J. (2002). The South African Community Epidemiology Network on Drug Use (SACENDU): Description, findings (1997-1999) and policy implications. Addiction, 97, 969-976.

Parry, C. D. H., Morojele, N. K., Saban, A., \& Flisher, A. J. (2004). Brief report: Social and neighbourhood correlates of adolescent drunkenness. A pilot study in Cape Town, South Africa. Journal of Adolescence, 27, 369-374.

Parry, C. D. H., Myers, B., Morojele, N. K., Flisher, A. J., Bhana, A., Donson, H., \& Pluddemann, A. (2004). Trends in adolescent alcohol and other drug use: Findings from three sentinel sites in South Africa (1997-2001). Journal of Adolescence, 27, 429-440.

Pavot, W., \& Diener, E. (1993). Review of the satisfaction with life scale. Psychological Assessment, 5(2), 164-171. 
Pluddemann, A., Parry, C., Bhana, A., Harker, N., Potgieter, H., Gerber, W., \& Johnson, C. (2004). The South African Community Epidemiology Network on Drug Use (SACENDU). Research brief, Phase 16, 7(2).

Reber, A. S., \& Reber E. S. (2001). The Penguin dictionary of psychology (3rd ed.). London: Penguin.

Reddy, S. P., Panday, S., Swart, D., Jinabhai, C. C., Amosun, S. L., James, S., Monyeki, K. D., Stevens, G., Morojele, N. S., Omardien, R.G., \& van de Borne, H. W. (2002). Umthente Uhlaba Usamila: The South African national youth risk behaviour survey. Cape Town: South African Medical Research Council.

Richter, L., Panday, S., Emmett, T., Makiwane, M., du Toit, R., Brooks, H., Potgieter, C., Altman, M., \& Mukhara, M. (2006). Young people in South Africa: The status of youth report 2003 - Final report. Research commissioned by the Umsobomvu Youth Fund. Pretoria: Human Sciences Research Council.

Rocha-Silva, L. (1998). Substance use on the rise in South Africa. In Focus Forum, 5(3), 51-53.

Rocha-Silva, L., de Miranda, S., \& Erasmus, R. (1995). Alcohol, tobacco and other drug use among black youth. Pretoria: Human Sciences Research Council.

Rocha-Silva, L., Mokoko, E., \& Malaka, D. (1998). Drug use in South Africa. Pretoria: Human Sciences Research Council.

Schwartz, R. C., \& Stack, S. D. (2003). Screening and assessing adolescent substance abuse: A primer for counsellors. Journal of Addictions \& Offender Counselling, 24(1), $23-24$.

Shiel, P. J. (1999). Predictor variables of adolescent risk-taking behaviour. Unpublished master's thesis, University of Pretoria, South Africa.

Simon, D. (1998). Drugs, development and the post-apartheid transition in South Africa. Urban Health and Development Bulletin, 1(4), 35-39.

Stoelb, M. (1998). A process model for assessing adolescent risk for suicide. Journal of Adolescence, 21, 359-370.

Sutherland, I., \& Shepherd, J. P. (2001). Social dimensions of adolescent substance use. Addiction, 96, 445-458.

Tarter, R. E. (2002). Etiology of adolescent substance use: A developmental perspective. The American Journal on Addictions, 11, 171-191. 